\title{
The promise and the problems of enzyme technology
}

\section{Peter Dunnill}

Immobilized Enzymes in Analytical and Clinical Chemistry: Fundamentals and Applications. Chemical Analysis, Vol.56. By P.W. Carr and L.D. Bowers. Pp.460. ISBN 0-471-04919-0. (Wiley: 1980.) £31.10, \$63.20. Immobilized Enzymes: An Introduction and Applications in Biotechnology. By M.D. Trevan. Pp.138. ISBN 0-471-27826-2. (Wiley: 1980.) £8.75, $\$ 24.10$.

THE great development which the field of immobilized enzyme technology has undergone is reflected in quite different ways in these two books. Ten years ago the field was so specialized that there was little need for a simple introductory text, but Michael Trevan's slim volume is published at a time when many people, including teachers, industrialists and even investors, need 10 know about the implications of enzyme technology, and have some grasp of the underlying principles. Equally, a subject such as the use of immobilized enzymes in analysis, which a decade ago warranted only a review chapter, now not only justifies a book, but Professors Carr and Bowers have had to add supplementary reference lists to deal with the outpouring of recent papers.

Dr Trevan divides his introductory account into three main parts. The first, dealing with the techniques of immobilization and its consequences for enzyme activity, is noteworthy for its clear and readable explanation of immobilized enzyme kinetics and the consequences of diffusional effects. The second reviews the current and potential applications of immobilized enzymes. This survey is comprehensive, but even more lead references would help to avoid any frustration in readers who wish to pursue more detailed information. The final section reflects renewed interest in the relationship between artificially immobilized enzymes and the functioning of enzymes which are fixed spatially within the cell, for example by association with membranes. Though the models which can be constructed to mimic active transport and other cellular processes are as yet crude, they point the way to exciting scientific developments. Introducing the possible industrial consequences of multi-enzyme catalysis to biochemistry students at the earliest possible stage could help to knit further the scientific and technological aspects of the subject.

The monograph by Carr and Bowers is a contribution to a series on analytical chemistry and sets out to provide a selfcontained explanation of the use of immobilized enzymes in analytical and clinical chemistry. Therefore il deals with basic enzymology and enzyme kinetics, and with appropriate immobilization techniques. Like Trevan's book, a sub- stantial part of the volume has to be devoted to the kinetics of immobilized enzymes, since a grasp of this aspect is central to interpretation of analytical results; however, as one would expect, the treatment is of greater depth and rigour, and this extends to the discussion of individual analytical methods where the focus is rightly on the underlying principles. The authors accept that immobilized systems have not made as much headway as expected, given the potential benefits in terms of reduced costs and increased accuracy. They ascribe this in part to the additional complexity of the two-phase enzyme-reagent system and to the need for a new method to show substantial advantages in order to displace established practice: anyone who has observed the pressures on hospital analytical facilities will know that introducing untried innovations is unlikely to be popular. However, the book's discussion of the strengths and weaknesses of immobilized enzymes for analysis should provide a firm foundation for the development of commercial systems.

Both books succeed in achieving their very different objectives and are valuable additions to the growing library of enzyme technology literature.

Peter Dunnill is Reader in Biochemical Engineering at University College, University of London.

\section{Pollution assessment: objectives and means}

\section{F. Moriarty}

Biological Monitoring for Environmental Effects. Edited by Douglas L. Worf. Pp.225. ISBN 0-669-03306-5. (Lexington Books: 1980.) £15.50, \$22.95.

THIS is the report of a conference and workshop held in North Carolina early in 1978. It was an all-American affair, and there is a lot of detail in the 22 papers that will not be of much interest to readers from other countries. Nevertheless, this book does raise several themes of general relevance for those who are concerned with monitoring the environmental effects of pollutants.

Biological monitoring was taken to mean the use of biota both to monitor the structure and function of ecosystems, and to measure the amount and distribution of contaminants. Several participants were concerned that current and impending regulations rely solely on chemical and physical measurements: the possibilities of, and need for, biological monitoring are not appreciated. Cairns and Dickson both emphasized that control of pollution by the "best applicable technology" is inappropriate, because it ignores the environment's capacity to deal with waste materials, and can result, in different circumstances, in either too much or too little protection of the environment.

A linked theme is the question of objectives. An adequate monitoring scheme cannot be designed until precise objectives have been stated. Weber discusses one aim of the Federal Water Pollution Control Act, to restore and maintain the biological integrity of the nation's waters. Biological integrity is not explicitly defined, and unless it means that ecosystems should remain in, or be restored to, their pristine condition, it is a nebulous concept that does not suggest objectives for monitoring. In practice, it is taken to mean fish, shellfish, wildlife and the effects of pollutants on the diversity, productivity and stability of communities of indigenous aquatic organisms. Osburn refers to the National Environmental Policy Act, which clearly accepts values such as aesthetic and cultural pleasure, and which can be translated into clear objectives.

Hirsch emphasizes that human impact on wildlife is synonymous with effects on ecosystems, discusses the scientific problems and then lists three institutional difficulties: lack of assured long-term funds for relevant ecological research, inadequate communication between administrators and ecologists, and lack of a focal point for expertise on ecological problems.

Other papers describe a range of techniques for monitoring in aquatic and terrestrial habitats, and the last five summarize the conclusions of five workshops. Current research is criticized for producing too many data of uncertain relevance; in addition more taxonomic skills are needed and a better ability to communicate scientific data 10 administrators. Research is also hampered by academic and administrative arrangements that militate against the development of multidisciplinary teams.

Several recent books have dealt with various aspects of monitoring. The range of topics in this book is limited, and much of the detail is of relatively parochial interest, but the problems encountered have a much wider relevance.

F. Moriarty is a Principal Scientific Officer at Monks Wood Experimental Station, Institute of Terrestrial Ecology, Abbots Ripion, Huntingdonshire. 